\title{
Electron Beam Exposure of Thermal Control Paints on Carbon-Carbon and Carbon-Polyimide Composites
}

\author{
Donald A. Jaworske \\ NASA Glenn Research Center, 21000 Brookpark Rd., Cleveland, OH 44135, USA \\ 216-433-2312,Donald.A.Jaworske@.nasa.gov
}

\begin{abstract}
Carbon-carbon and carbon-polyimide composites are being considered for use as radiator face sheets or fins for space radiator applications. Several traditional white thermal control paints are being considered for the surface of the composite face sheets or fins. One threat to radiator performance is high energy electrons. The durability of the thermal control paints applied to the carbon-carbon and carbon-polyimide composites was evaluated after extended exposure to $4.5 \mathrm{MeV}$ electrons. Electron exposure was conducted under argon utilizing a Mylar ${ }^{\mathrm{TM}}$ bag enclosure. Solar absorptance and infrared emittance was evaluated before and after exposure to identify optical properties degradation. Adhesion of the paints to the carbon-carbon and carbon-polyimide composite substrates was also of interest. Adhesion was evaluated on pristine and electron beam exposed coupons using a variation of the ASTM D-3359 tape test. Results of the optical properties evaluation and the adhesion tape tests are summarized.
\end{abstract}

Keywords: Solar absorptance, infrared emittance.

PACS: 78.20.Ci

\section{INTRODUCTION}

Radiator surfaces are characterized by the two optical properties of solar absorptance and infrared emittance. For radiators operating in the presence of solar energy, the preferred radiator surface is white, often a ceramic coating having the combined properties of low solar absorptance and high infrared emittance. Radiators are typically composed of a sandwich structure, utilizing honeycomb sandwiched between two face sheets, or may be composed of fins extending from a central fluid carrying pipe. Advanced materials are of interest for space radiator face sheet applications. Carbon-carbon and carbon-polyimide composites made from high thermal conductivity pitch-based graphite fibers are of particular interest owing to their light weight and attractive thermal properties. These pitchbased graphite fibers used in concert with a suitable matrix material also offer the ability to tailor the coefficient of thermal expansion of the composite to match the coefficient of thermal expansion of the underlying components. For missions of long duration to the gas giants, electron exposure is expected to be significant. Hence, electron exposure testing of composite coupons coated with thermal control coatings was of interest.

Irradiation of composite samples with electrons can been accomplished in a variety of ways. Paillous and Pailler (1994) utilized asymmetric irradiation, three successive electron energies to reproduce a particular dose profile. Electrons were delivered from a Van de Graff accelerator. In addition, they utilized uniform irradiation, a single irradiation with $2 \mathrm{MeV}$ electrons to produce a dose profile uniform through the sample thickness. Thermal cycling from $-100{ }^{\circ} \mathrm{C}$ to $+100{ }^{\circ} \mathrm{C}$ yielded a combined effect of radiation and thermal cycling that caused chain scission which plasticized the composite matrix at elevated temperatures and embrittled the matrix at low temperatures causing microcracking. Milkovich, Herakovich and Sykes (1986) utilized a dynamitron to irradiate composite samples with $1 \mathrm{MeV}$ electrons, equivalent to 30 years in GEO. Electron radiation degraded the epoxy matrix yielding similar results where the irradiation plasticized the epoxy of the composite at high temperatures with the evolution of some volatiles. They observed the increased plasticity in stress-strain curves having a broad rubbery range that reflected the presence of radiation induced degradation products. They further observed a lowered glass transition temperature, suggesting a degraded network structure in the epoxy. At low temperatures, the epoxy in the composites was found to be embrittled. 
Recently, to address testing of materials for missions to the gas giants, a three bin concept, i.e. a group fluence approach, was advocated by Willis (2004). Figure 1 summarizes the bin concept, highlighting electrons and protons for geosynchronous Earth orbit (GEO) and the Jupiter moon Europa. Bin 1 represents low energy electrons having a high flux and is utilized as a test for screening the durability of materials. Bin 2 has more energetic electrons having a lesser flux. Only those materials successfully passing the screening test are subjected to Bin 2 testing. Bin 3 has the most energetic electrons with the least flux, and is the most expensive of the Bin testing regimen. The philosophy adopted here was to expose carbon-carbon and carbon-polyimide composite coupons painted with various white thermal control paints to a total surface dose of approximately $1000 \mathrm{MR}$ (megarad). It should be noted that surface doses are expressed as thermal equivalents; one rad equals $100 \mathrm{erg} / \mathrm{gram}$, or $0.01 \mathrm{Joules} / \mathrm{Kg}$, and that actual dose is the energy deposited by electrons that are successfully absorbed within a specific mass or volume. Actual dose was not measured. Such a measurement would have required a dosimeter to identify the electrons not absorbed by the samples in order to calculate actual dose by difference. However, the surface dose was calculated to be $1,010 \mathrm{MR}$ and the electron fluence at the surface was $6.12 \times 10^{15}$ electrons $/ \mathrm{cm}^{2}$ at an energy of $4.5 \mathrm{MeV}$.

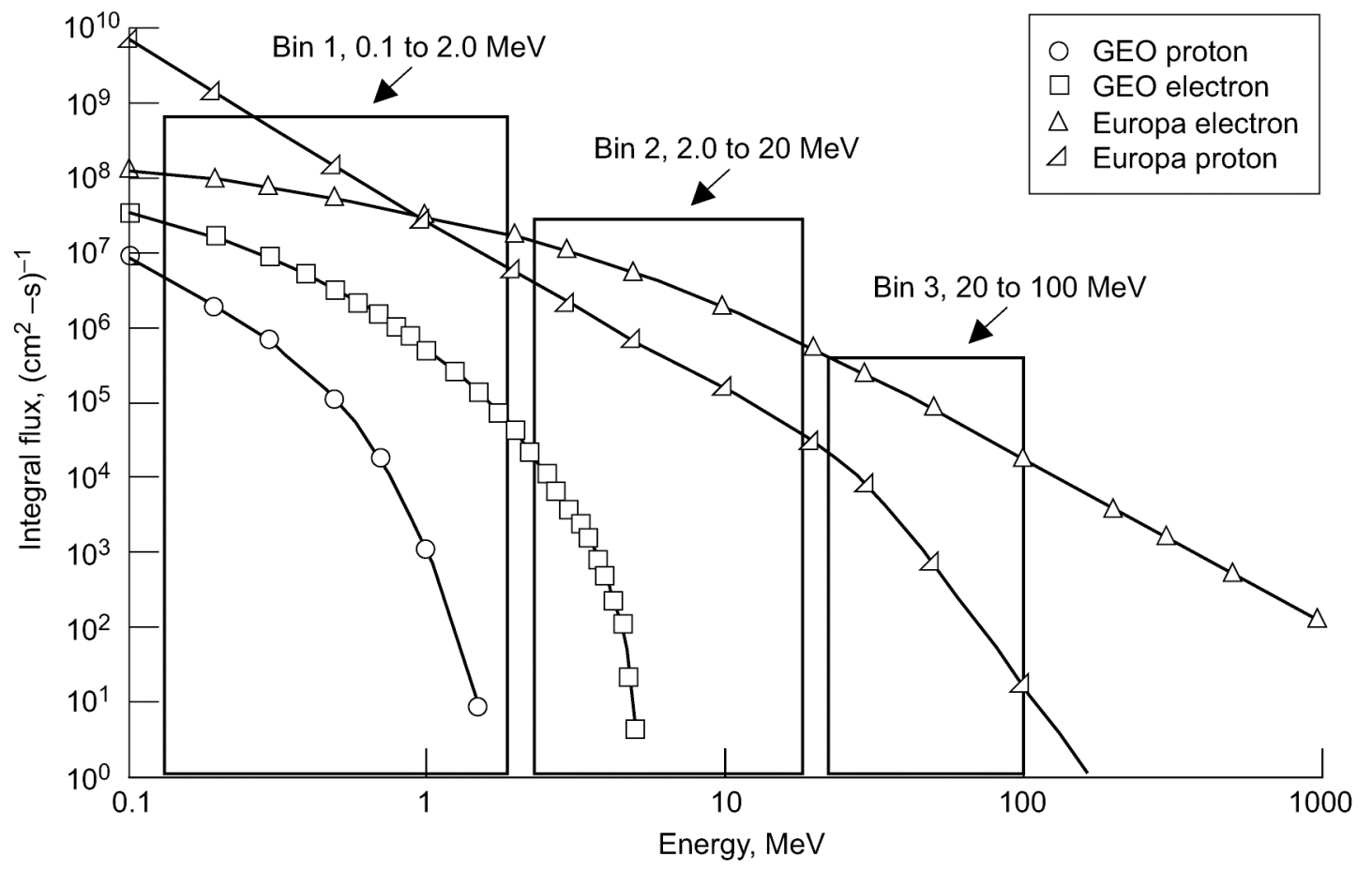

FIGURE 1. Bin Concept for Electron Exposure of Samples (Willis, 2004).

The use of this approach simulates total mission fluence, hence, there is no need to extrapolate exposure to total fluence. Ionizing radiation testing may have a role in polymer damage (chain scission, embrittlement, outgassing) and thermal control paint discoloration. Hence, damage mechanisms are expected to include de-bonding of paint from the polymer surface, and a potential change in solar absorptance due to darkening. This paper summarizes the screening of several candidate carbon-carbon and carbon-polyimide composites coated with various white thermal control paints.

\section{MATERIALS AND METHODS}

Two carbon-carbon composites and one carbon-polyimide composite were selected for testing. Both types of carbon-carbon composites were prepared with pitch-based P-120 graphite fibers. One set of carbon-carbon composites were made by C-CAT (Carbon-Carbon Advanced Technologies), Inc., Fort Worth, TX by a resin infiltration and pyrolysis process, designated here as C-CAT. Another set of carbon-carbon composites were made by Goodrich Corp., Santa Fe Springs, CA by high temperature pitch impregnation and pyrolysis followed by carbon 
densification using a chemical vapor infiltration (CVI) process, designated here as Goodrich. The composites were machined into $5 \mathrm{~cm} \times 5 \mathrm{~cm}$ pieces, cleaned in acetone using an ultrasonic bath, and dried in an oven before the application of thermal control coatings. The carbon-polyimide was manufactured at Glenn Research Center as part of an ongoing research effort and utilized PAN-based T-650 fibers, designated here as carbon-polyimide composite.

Three thermal control paints were considered. One thermal control paint, manufactured by AZ Technology, Huntsville, AL, was a non conductive formulation using zinc oxide pigment in a potassium silicate binder, designated AZ-93. As part of the deposition process for this paint, an epoxy primer was utilized to form the interface between composite and paint. The second paint was manufactured by Alion, Chicago, IL, and was a formulation using a zinc-ortho-titanate pigment in a potassium silicate binder, designated YB-71P. The surface of each composite painted with YB-71P was purposely abraded utilizing a proprietary method to promote adhesion. The third paint was also manufactured by Alion, and was another formulation using a zinc oxide pigment in a potassium silicate binder, designated Z-93-C55. This paint included a conductive oxide dopant to provide some electrical conductivity. Again, the surface of the composite coupons was abraded prior to painting to promote adhesion. All three thermal control paints were applied utilizing a spray on technique.

Solar absorptance was evaluated utilizing a Perkin-Elmer Lambda-19 spectrophotometer equipped with a $15 \mathrm{~cm}$ diameter integrating sphere. This spectrophotometer is a dual beam system that purposely negates subtle changes in light intensity from the lamp sources. Total reflectance was obtained in the wavelength range of 250 to $2500 \mathrm{~nm}$, subtracted from unity, and weighted with respect to the air mass zero solar spectrum to yield solar absorptance. Solar absorptance, as calculated with this method, is temperature independent. Infrared emittance was evaluated utilizing a Surface Optics Corporation SOC-400t portable infrared reflectometer equipped with barrel optics. Total reflectance was obtained in the wavelength range of 2 to 25 microns, subtracted from unity, and weighted with respect to the blackbody spectrum at room temperature to yield infrared emittance. Although room temperature emittance values are utilized here for comparison, this method can also be used for identifying emittance at temperatures other than room temperature, providing the appropriate blackbody spectrum is used during the weighting step in the calculation. That is, infrared emittance, as calculated with this method, is temperature dependent.

Electron exposure was accomplished at E-beam Services, Lebanon, $\mathrm{OH}$, utilizing an industrial accelerator capable of providing electron energies up to $10 \mathrm{MeV}$. At such high energies, the electron beam passes from its vacuum system through a titanium window and is rastored over samples placed on a conveyer system under the beam. For this study, 4.5 MeV electrons were used. Repeated passes under the beam were arranged to yield a total exposure of 1010 MR. The carbon-carbon and carbon-polyimide composites were mounted on an aluminum plate, and were bagged in aluminized Mylar ${ }^{\mathrm{TM}}$ under inert gas. The bag was changed periodically during the course of electron beam exposure. Coupon temperature was monitored through the use of temperature strips placed on the aluminum plate near the coupons. The epoxy interface layer utilized on some samples limited the upper use temperature for these coupons to $177^{\circ} \mathrm{C}$, and the polyimide matrix in some samples limited the upper use temperature for these coupons to $277^{\circ} \mathrm{C}$. The temperature strips utilized during electron beam exposure indicated that the maximum temperature did not exceed $125^{\circ} \mathrm{C}$.

Adhesion testing was conducted on pristine and electron beam-exposed samples. The adhesion testing was based on a variation of ASTM D-3359 test method A, the X-cut tape test. This variation was used based on past experience in evaluating Z-93-P thermal control paint. In test method A, a $5 \mathrm{~cm}$ " $\mathrm{X}$ " is scribed onto the coupon with a scalpel, a $2.54 \mathrm{~cm}$ wide pressure sensitive standard tape is applied on top of the " $\mathrm{X}$ " using a rubber eraser. The tape is then removed rapidly within 90 seconds of its application, pulling back at an angle as close to $180^{\circ}$ as possible. A six point scale is provided in the standard to rate the adhesion. Table 2 summarizes the rating scale. The variation used here utilized two $5 \mathrm{~cm}$ parallel lines scribed onto the coupon approximately $1.5 \mathrm{~cm}$ apart. A scalpel was used, cutting through the paint to the substrate in a single cut. The tape was applied normal to the two scribed lines with four passes of a rubber roller. The tape was removed rapidly after 30 seconds of its application by pulling back at an angle as close to $90^{\circ}$ as possible. The tape for the tape test was a 3M 250 paper backed masking tape, the same type of tape used in previous evaluation of Z-93-P thermal control paint.

TABLE 1. Rating Scale for the Adhesion Test.

Rating Description


$5 \mathrm{~A}$

$4 \mathrm{~A}$

$3 \mathrm{~A}$

$2 \mathrm{~A}$

$1 \mathrm{~A}$

$0 \mathrm{~A}$

No peeling or removal

Trace peeling or removal along incisions

Jagged removal along incisions up to $1.8 \mathrm{~mm}$ on either side

Jagged removal along most of incisions up to $3.2 \mathrm{~mm}$ on either side

Removal from most of the area under the tape

Removal beyond the area under the tape

\section{RESULTS AND DISCUSSION}

The as-painted coupons appeared to have good paint coverage, with the white paint samples appearing pale white for the Z-93-C55 to bright white for the AZ-93 and YB-71P. Paint thickness was estimated to be $0.08 \mathrm{~mm}$. Photographs of each sample were obtained next to pristine sister samples to show the change in appearance and are summarized in Figure 2.
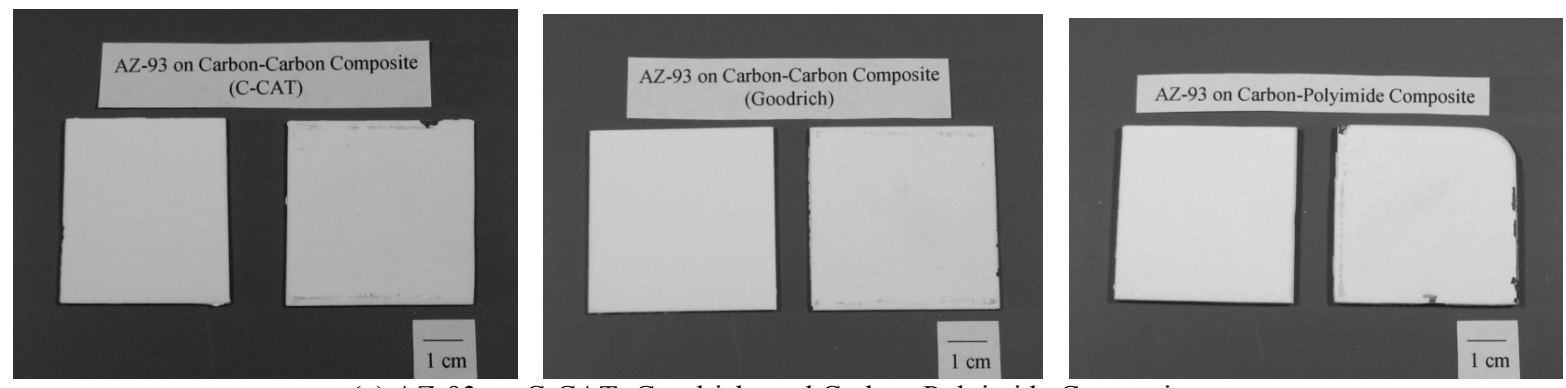

(a) AZ-93 on C-CAT, Goodrich, and Carbon-Polyimide Composites.
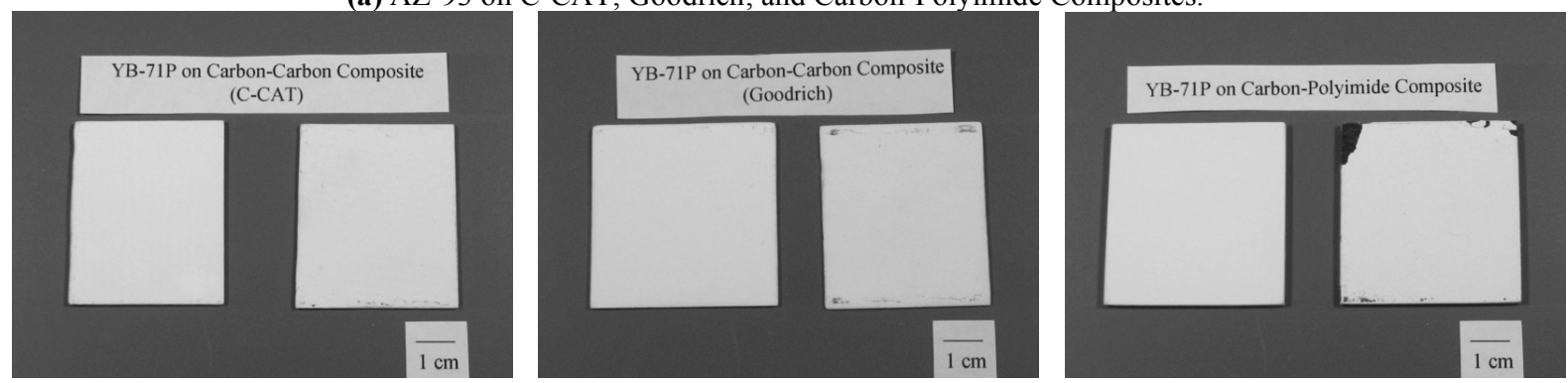

(b) YB-71P on C-CAT, Goodrich, and Carbon-Polyimide Composites.
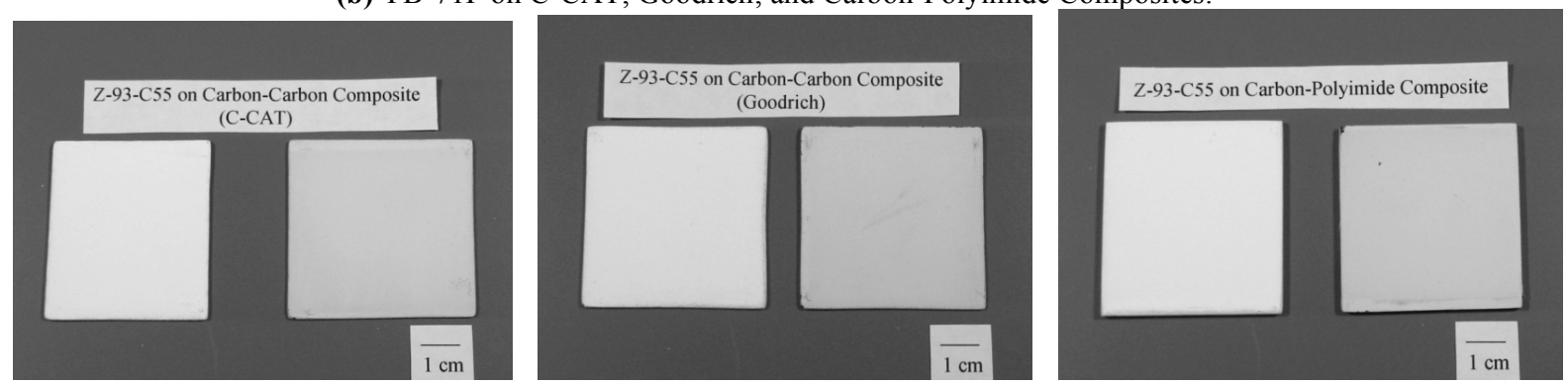

(c) Z-93-C55 on C-CAT, Goodrich, and Carbon-Polyimide Composites.

FIGURE 2. Photographs of Pristine (Left) and Exposed (Right) Coupons After Electron Beam Exposure.

Each vendor reported that applying their paint to the composites was difficult, particularly the carbon-polyimide composite. The post electron exposure appearance varied. The AZ-93 and YB-71P coupons showed a similar good coverage after electron exposure, with some minor cracking. The Z-93-C55 samples appeared noticeably pale, darker than their pristine counterparts. One Z-93-C55 sample had a streak down the center. 
The initial solar absorptance for each candidate paint, along with its post electron beam exposure solar absorptance, is summarized in Table 2. Within a given series of painted coupons, values obtained before exposure indicate the uniformity in solar absorptance, with AZ-93 having the most narrow distribution of values and YB-71P having the widest distribution of values. After electron exposure, the solar absorptance values of AZ-93 and YB-71P show a slight increase in solar absorptance and Z-93-C-55 shows a substantial increase in solar absorptance. Initial infrared emittance for each candidate paint, along with its post electron beam exposure infrared emittance, is summarized in Table 3. Again, within a given series of painted coupons, values obtained before exposure indicate the uniformity of infrared emittance, with all three paints exhibiting a narrow distribution of values. After electron exposure, the infrared emittance values showed little change, with all three paints exhibiting a slight decrease in infrared emittance.

TABLE 2. Solar Absorptance, Before and After Electron Beam Exposure.

\begin{tabular}{lccc}
\hline \multicolumn{1}{c}{ Paint } & C-CAT & Goodrich & Carbon-Polyimide \\
\hline AZ-93 & $0.119 / 0.133$ & $0.118 / 0.147$ & $0.121 / 0.159$ \\
YB-71P & $0.118 / 0.178$ & $0.087 / 0.150$ & $0.128 / 0.160$ \\
Z-93-C55 & $0.145 / 0.292$ & $0.152 / 0.313$ & $0.144 / 0.288$ \\
\hline
\end{tabular}

TABLE 3. Infrared Emittance, at $27^{\circ} \mathrm{C}(300 \mathrm{~K})$, Before and After Electron Exposure.

\begin{tabular}{lccc}
\hline \multicolumn{1}{c}{ Paint } & C-CAT & Goodrich & Carbon-Polyimide \\
\hline AZ-93 & $0.955 / 0.949$ & $0.954 / 0.950$ & $0.955 / 0.950$ \\
YB-71P & $0.929 / 0.922$ & $0.934 / 0.930$ & $0.927 / 0.923$ \\
Z-93-C55 & $0.950 / 0.946$ & $0.949 / 0.945$ & $0.958 / 0.951$ \\
\hline
\end{tabular}

Adhesion testing, was conducted on the samples after exposure to the electron beam. Additional adhesion testing was conducted on pristine sister samples for comparison. The results of the adhesion testing are summarized in Table 4. The specimens having the epoxy interface layer were found to have only trace peeling along the parallel incisions with very little material actually removed. The trace peeling along the parallel incisions was remarkably similar in both the pristine case and the after electron exposure case, suggesting little change in the bonding at the interface as a consequence of the electron exposure. Of the three types of paint studied here, the coupons having the epoxy interface represented the best thermal control paint results to-date. All of the carbon-carbon and carbonpolyimide specimens having an interface that was purposely roughened and painted with either the YB-71P or Z-93C55 had poor adhesion initially and total failure after electron exposure. In some cases, the paint simply lifted off the substrate with substantial removal beyond the area under the tape.

TABLE 4. Adhesion Test Results, Pristine and After Electron Beam Exposure.

\begin{tabular}{llll}
\hline \multicolumn{1}{c}{ Paint } & C-CAT & Goodrich & Carbon-Polyimide \\
\hline AZ-93 & $4 \mathrm{~A} / 3 \mathrm{~A}$ & $4 \mathrm{~A} / 4 \mathrm{~A}$ & $4 \mathrm{~A} / 4 \mathrm{~A}$ \\
YB-71P & $0 \mathrm{~A} / 0 \mathrm{~A}$ & $2 \mathrm{~A} / 0 \mathrm{~A}$ & $0 \mathrm{~A} / 0 \mathrm{~A}$ \\
Z-93-C55 & $1 \mathrm{~A} / 0 \mathrm{~A}$ & $3 \mathrm{~A} / 0 \mathrm{~A}$ & $0 \mathrm{~A} / 0 \mathrm{~A}$ \\
\hline
\end{tabular}

Visual observations were made at $200 \mathrm{MR}, 400 \mathrm{MR}$, and $773 \mathrm{MR}$, during the re-bagging activities between electron exposures. At $200 \mathrm{MR}$ and $400 \mathrm{MR}$, the samples seemed fine. At $773 \mathrm{MR}$, some darkening was beginning to show in the Z-93-C55 coupons.

The optical properties and adhesion test results both suggest that the AZ-93 with epoxy interface offers the best thermal control paint tested here, for both carbon-carbon composites and the carbon-polyimide composite. However, it should be noted that the epoxy interface limits the upper operating temperature of the system to the upper operating temperature of the epoxy. Unfortunately, this upper limit is estimated to be $177^{\circ} \mathrm{C}$, which is lower 
than the desired operating temperature of future space radiator face sheet applications. Hence, more work is needed to identify high temperature interface layer candidates.

\section{ACKNOWLEDGMENTS}

The author thanks Angel Garnica, Jet Propulsion Laboratory, for his watchful guidance at E-Beam services during the re-bagging of coupons, Paul Willis, Jet Propulsion Laboratory, for his helpful discussions, and Edward Maslowski, Analex Corporation, for his help and guidance in preparing and transporting the coupons.

\section{CONCLUSION}

Much of the work done in the area of advanced radiator development has centered around developing new design concepts that incorporate advanced radiator materials, such as carbon-carbon and carbon-polyimide composites. However, much work still remains in understanding the application of traditional thermal control paints to these substrates and the interaction of these thermal control paints with carbon-carbon and carbon-polyimide composites, especially at the interface. Optical properties evaluation of coupons before and after electron beam exposure to 1010 MR indicates that the surfaces hold up to varying degrees, with some paints exhibiting little change in performance while others darken noticeably. Adhesion testing reveals that thermal control paints developed for use on aluminum and other metals are not suitable for use on bare composites. Utilizing an epoxy interface layer helps, however, the epoxy places a temperature limit on the system.

\section{REFERENCES}

ASTM International, Standard Test Methods for Measuring Adhesion by Tape Test, ASTM D-3359, ASTM International, West Conshohocken, PA, pp. 1-7, 2002.

Milkovich, S.M., Herakovich, C.T., and Sykes, G.F., "Space Radiation Effects on the Thermo-Mechanical Behavior of Graphite-Epoxy Composites,” J. of Composite Materials, 20, 579-593, (1986).

Paillous, A., and Pailler, "Degradation of Multiply Polymer-Matrix Composites Induced by Space Environment," Composites, 25 (4), 287-295, (1994).

Willis, P., NASA Jet Propulsion Laboratory, Pasadena, CA, Personal Communication, 2004. 